\title{
Beyond Boveri
}

DOI:

10.1038/nrc1903

\section{URLs}

p16 $6^{\text {INK } 4 a}$

http://www.ncbi.nlm.nih.gov/

entrez/query.fcgi?db=gene\&c

$\mathrm{md}=$ Retrieve\&dopt $=$ Graphics\&

list_uids=1029

CDK2

http://www.ncbi.nlm.nih.gov/ entrez/query.fcgi?db=gene\&c $\mathrm{md}=$ Retrieve\&dopt=full report\&list_uids $=1017$

p21

http://www.ncbi.nlm.nih.gov/ entrez/query.fcgi?db=gene\&c $\mathrm{md}=$ Retrieve $\& \mathrm{dopt}=$ full report\&list_uids $=1026$
The gain or loss of whole chromosomes (aneuploidy) is the most frequently identified genomic abnormality in cancer, but the mechanism through which this arises is poorly understood. Now, Thea Tlsty and colleagues show that the generation of aneuploid daughter cells can result from the generation of too many centrosomes through a pathway that involves the tumour suppressor p16 $6^{\text {INK4a }}$.

The classic hypothesis by Theodor Boveri, published in 1914, proposed that aneuploidy results from an increase in the number of centrosomes - organelles that organize the poles of the mitotic spindle. Tlsty and colleagues investigated this further through the use of a variant population of primary human mammary epithelial cells (vHMECs) that accumulate additional centrosomes with continued population doublings and become aneuploid. They showed that the additional centrosomes are not due to polyploidy, and that inhibiting DNA synthesis with hydroxyurea (HU) led to the acquisition of too many centrosomes in these cells, which indicates that centrosome duplication and DNA replication are uncoupled.

The loss of $\mathrm{p} 16^{\mathrm{INK} 4 \mathrm{a}}$ expression is a distinguishing characteristic of vHMECs, so could this have a causal role in the acquisition of additional centrosomes? RNA interference with the mRNA that encodes $\mathrm{p} 16^{\mathrm{INK4a}}$ in normal HMECs (which express p16 ${ }^{\text {INK4a }}$ and do not accumulate additional centrosomes) resulted in an increased proportion of HMECs with additional centrosomes after exposure to HU. Conversely, transfection of vHMECs with a plasmid that expresses wild-type $\mathrm{p} 16^{\mathrm{INK4a}}$ inhibited the generation of additional centrosomes following exposure to HU. So, the acquisition of additional centrosomes because of the loss of p $16^{\text {INK4a }}$ activity most probably explains the production of aneuploid daughter cells.

Closer examination of the additional centrosomes by immunocytochemistry revealed that, although still functional, a statistically significant fraction contained only one centriole. This indicates that these additional centrosomes are generated by centriole-pair splitting and that $16^{\mathrm{INK4a}}$ must therefore prevent this during $\mathrm{S}$ phase.

Cyclin-dependent kinase 2 (CDK2) is known to regulate DNA synthesis and centrosome duplication, so does the $\mathrm{p} 16^{\mathrm{INK4a}}$ pathway involve CDK2? Tlsty and colleagues showed that inhibiting CDK2 activity prevented the acquisition of additional chromosomes in vHMECs treated with HU. Furthermore, their data indicate that $\mathrm{p} 16^{\mathrm{INK} 4 \mathrm{a}}$ regulates CDK2 activity by interacting directly with the cyclin-dependent kinase inhibitor $\mathrm{p} 21$.
So, p16 $6^{\text {INK4a }}$ has a key function in centrosome biology, acting through CDK2 to prevent centriole-pair splitting and to couple the DNA-replication and centrosome-duplication cycles. Loss of $\mathrm{p} 16^{\mathrm{INK} 4 \mathrm{a}}$ could give cells a proliferative advantage under stress conditions: the transient inhibition of DNA synthesis would lead to the acquisition of additional centrosomes and, consequently, the generation of aneuploid daughter cells. This disruption in gene dosage might provide the necessary pro-proliferation and anti-apoptotic mechanisms required for tumorigenesis.

Rebecca Robey, Assistant Editor, Oncogene

ORIGINAL RESEARCH PAPER McDermott, K.

M. et al. p16INK4a prevents centrosome dysfunction and genomic instability in primary cells. PLoS Biol. 4, 350-365 (2006)

WEB SITE

Thea Tlsty's home page: http://cc.ucsf.edu/ people/tlsty_thea.html 